*These authors contributed equally to this work

Address for Correspondence:

Dr. Jie-mingQu, MD \& PhD, Rui Jin

Hospital, Shanghai Jiao Tong University

School of Medicine, No. 197, Rui Jin Er

Road,Shanghai200025, China

Email: jmqu0906@163.com

\begin{tabular}{|l|}
\hline Access this article online \\
\hline Website: \\
www.intern-med.com \\
\hline DOI: \\
10.2478/jtim-2018-0006 \\
\hline Quick Response Code: \\
\hline \\
\hline \\
\\
\hline
\end{tabular}

\title{
Contemporary situation of community- acquired pneumonia in China: A systematic review
}

\author{
Ying-gang Zhu ${ }^{1, *}$, Xiao-dan Tang ${ }^{1, *}$, Yun-tao $\mathbf{L u}^{1}$, Jing Zhang ${ }^{2}$, Jie-ming $\mathbf{Q u}^{3}$ \\ 'Department of Pulmonary Disease, Huadong Hospital, Fudan University, Shanghai 200040, China; \\ 2Department of Pulmonary Disease, Zhongshan Hospital, Fudan University, Shanghai 200032, China; \\ ${ }^{3}$ Department of Respiratory and Critical Care Medicine, Ruijin Hospital, \\ Shanghai Jiao Tong University School of Medicine, Shanghai 200025, China
}

\section{ABSTRACT}

The prevalence and microbial pattern reported for Community-acquired pneumonia (CAP) differ considerably and contemporary situation remains changing over time. We therefore searched both international and domestic databases for relevant references and pooled incidence of CAP and etiological distribution were estimated separately between children and adults groups. The results showed that CAP remained a major public health issue in China, with a relatively higher incidence than that reported in Western countries. Although pathogens were not detected in nearly half of patients, Mycoplasma pneumoniae remained to be the most frequently detected agent across age groups, the detection yield of which was lower than that reported from other countries. Notably, the incidence of influenza virus A in adults was almost four times higher than that in children while the prevalence of respiratory syncytial virus was much less common in adults than that in children. Despite some limitations, the value of this review, approaching to systematically review grey published data, is to sketch out the contemporary epidemiological and etiological situation of CAP in our country, which could be useful to help policymakers and clinicians make informed choices and to inspire future studies and surveillance.

Key words: community acquired pneumonia, epidemiology, etiology,antibiotics resistance

\section{INTRODUCTION}

Although community-acquired pneumonia (CAP) remains a common disease with high mortality and economic cost, the prevalence and microbial pattern reported for CAP differ considerably worldwide, depending on epidemiologic area, patient populations, and the extent and nature of the microbiologic techniques used. ${ }^{[1,2]}$ Knowledge of predominant microbial patterns in CAP, as a consensus, constitutes the basis for guiding the empirical antimicrobial treatment. ${ }^{[3,4]}$ Contemporary estimates of the nationwide incidence and microbiologic causes for CAP would be of great value. However, the last multi-center publication of etiology of CAP in Chinese urban population was almost a decade ago and situation remained changing over time. ${ }^{[5]}$ We, therefore, performed a systematic literature review from both international and domestic databases to summarize some of the most concerning questions in CAP such as incidence, etiological distribution and resistance to antibiotics in China.

\section{METHODS}

We searched PubMed,EMBASE and Web of Science as well as nationwide recognized databases such as WanFang (wanfangdata.com.cn), National Knowledge Infrastructure (cnki.net) and CQVIP (cqvip. com) for relevant studies published up toJan 2017. Given the aim of our review, we mainly focused on prevalence, pathogen detection rate, etiological distribution and antibiotic resistance. Pooled incidence of CAP and etiological distribution were estimated separately between children and adults groups. 


\section{RESULTS}

\section{Incidence}

Two hundred andsixty-nine publications were finally included with 154 publications for adults and 115 for children. The incidence of CAP ranged from 29.8 to 221.0 per 10,000 admissions. The proportion of elderly cases (65 years of age or older) among all CAP patients was $37.2 \% \pm 7.9 \%$. The trial with largest scale population (Guangdong, 2009) suggested that the incidence of CAPincreasedfrom $0.22 \%(8,677 / 3,893,800)$ in 2009 to $0.41 \%(19,947 / 4,839,766)$ in 2012 and mortality rate was highest among elderly patients (65 years of ageor older) $(4.70 \%, 224 / 4,763)$. The incidence was similar across genders and different seasons.

\section{Detection of Pathogens}

Overall, the pathogens detected yield among adults was $51.0 \% \pm 17.9 \%$ and that among children was $52.0 \%$ $\pm 18.1 \%$ (Figure 1). No significant differences of rate between adults and children were observed among different regions throughout the country (Northern, $49.8 \%$ vs. $55.0 \%$; Eastern, $47.7 \%$ vs. 51.4\%; Central \& Southern, $54.0 \%$ vs. $53.7 \%$; Western, $56.3 \%$ vs. $47.4 \%$ ).

The distribution of overall etiology was shown in Figure 2. Mycoplasmapneumoniae was the most common agent among adults, identified in $15.01 \%$ of patients, followed by Streptococcuspneumoniae in $11.68 \%$, Influenza virus $\mathrm{A}$ in $12.28 \%$ and fungi in $8.28 \%$. On the other hand, Mycoplasmapneumoniae was also the most common agent among children, identified in $19.16 \%$ of patients, followed by Respiratorysyncytial virus in $16.01 \%$, Streptococcuspneumoniae in $9.44 \%$ and Klebsiellapneumoniae in $7.77 \%$. Specifically, significant differences on the detected rate of Escherichiacoli and Pseudomonas aeruginosa were found between adults and children $(3.88 \% v$ s. $6.95 \%, P<0.05$ and $4.37 \%$ vs. $1.22 \%, P<0.05$, respectively).
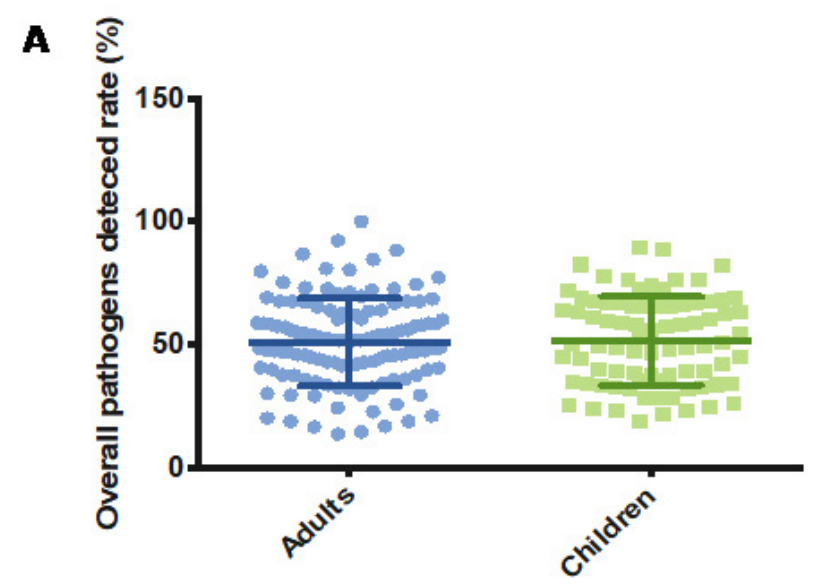

B
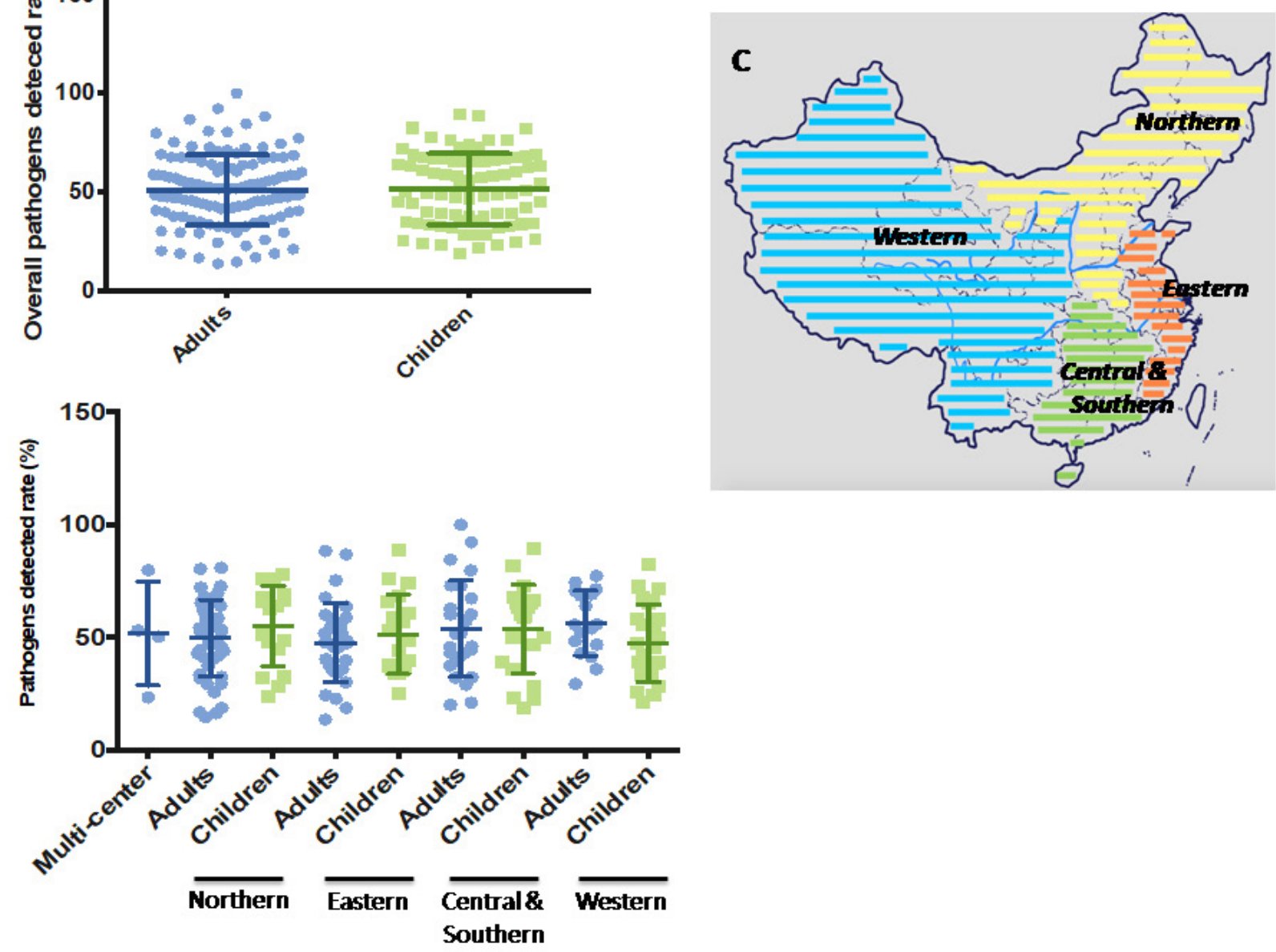

Figure 1: Overall pathogens detected yield among adults and children depending on regions. (A) Overall pathogens detected yield among adults and children; (B) Pathogens detected yield depending on designed regions; (C) Colors labeled on the map of China showed the designed areas in Panel C. Yellow (Northern area): provinces of Beijing, Tianjin, Heilongjiang, Jilin, Liaoning, Hebei, Henan, Shan(1)xi and Inner Mongolia. Red (Eastern area): provinces of Shanghai, Jiangsu, Zhejiang, Shandong, Anhui and Fujian. Green (Central \& Southernarea): provinces of Hubei, Hunan, Jiangxi, Guangdong, Guangxi and Hainan. Blue (Western area): provinces of Shan(3)xi, Sichuan, Chongqing, Guizhou, Yunnan, Ningxia, Gansu, Qinghai, Xinjiang and Tibet. 
The fungi were detected more commonly in adults than in children $(8.28 \%$ vs. $1.76 \%, P<0.01)$. The frequency of Influenza virus $A$ in adults was significantly higher than that in children $(12.28 \%$ vs. $3.73 \%, P<0.01)$ while the frequency of Respiratory syncytial virus (RSV) in children was significantly higher than that in adults $(16.01 \% \mathrm{vs}$. $1.24 \%, P<0.001)$.

\section{Antimicrobial susceptibility}

A total of 93 publications (50 in adults and 43 in children) submitted Streptococcuspneumoniaefor antimicrobial susceptibility testing. The proportions of intermediately/ fully resistant isolates to penicillin from adult and child patients were $43.33 \%$ and $55.68 \%$, respectively. However, no statistical difference was found between two groups.

\section{DISCUSSION}

Although several multi-center studies regarding nation-wide epidemiology and etiology of CAP have been published recently, ${ }^{[5-8]}$ the contemporary situation in reality remains attractive. The main reason results fromhuge publication biasbecause most Chinese clinical researchers have limited English writing skills, which means we probably failed to notice valuable data sealed within Chinese databases. The present review, therefore, is the first to systematically analyze several concerning questions on CAP from both international and domestic resources. The results, combined from all ages, revealed a relatively higher incidence (29.8 to 221.0 cases per 10,000 persons) of CAP than that reported among adults (24.8 to 26.7 cases per 10,000 adults) ${ }^{[1]}$ or children(15.7 to 22.5 cases per 10,000 children younger than 18 years old $)^{[2]}$ in USA. Despite of large scale of admission population, existing data mainly originated from developed urban areas with a more sophisticated hygienic system compared to rural areas, which means that hospitals, in those cities, were more accessible to the public. The other possibility wasthat theinfluence of immigrants from nearby counties seeking for better medical resourceswould contribute to this increasing morbidity in urban areas. However, those limited investigations were not sufficient to depict the whole figure of the country.

According to the previous studies, atypical pathogens, including M. pneumoniae, C. pneumoniae, and L. pneumophila, have been reported more frequently over time, responsible for 20 to $40 \%$ of cases of CAP. ${ }^{[5,9,10]}$ Although our combined data in China revealed M.pneumoniaeremained
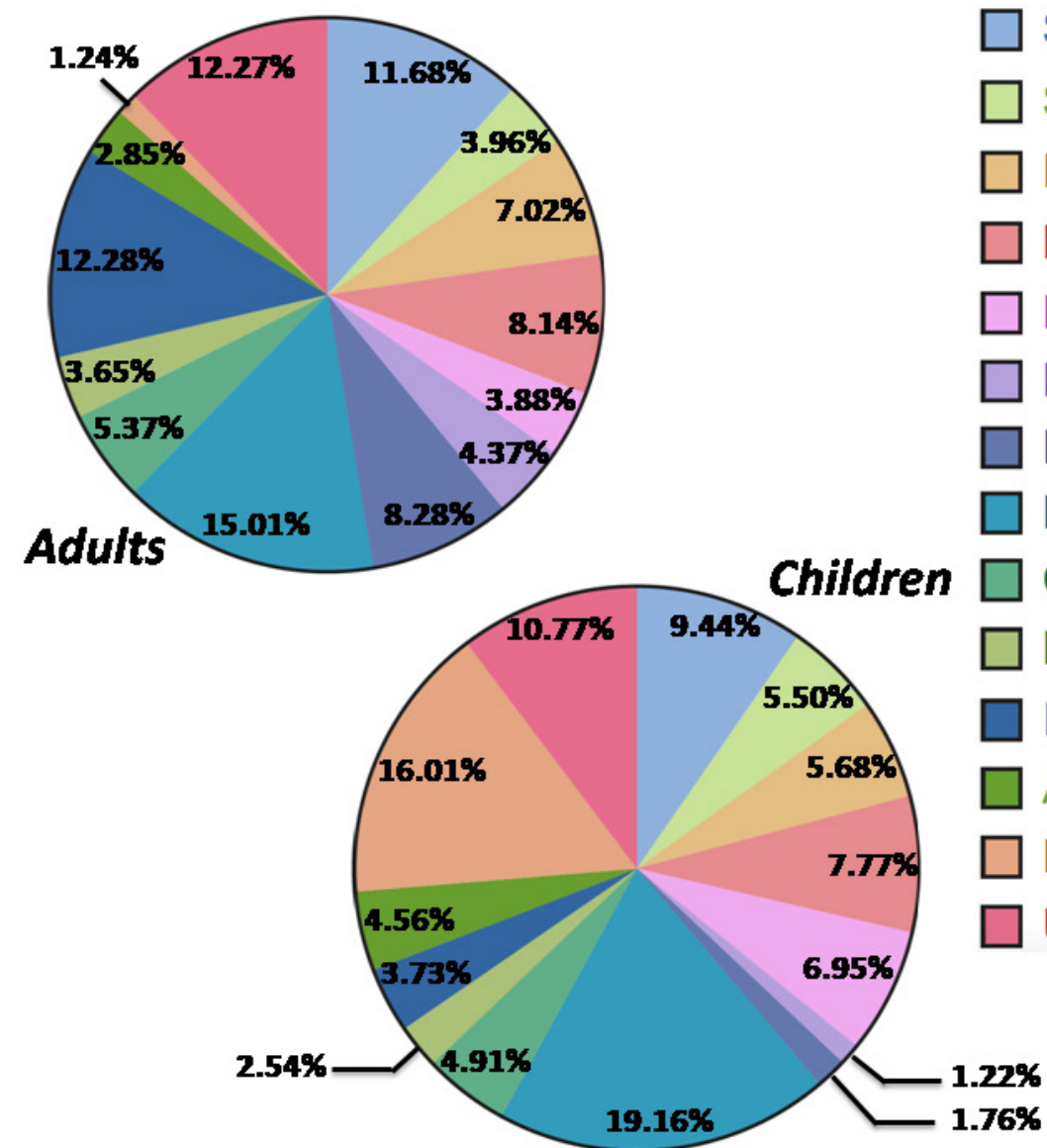

$\square$ Streptococcus pneumoniae

$\square$ Staphylococcus aureus

Haemophilus influenzae

Klebsiella pneumoniae

$\square$ Escherichia coli

Pseudomonas aeruginosa

Fungi

Mycoplasma pneumoniae

Chlamydia pneumoniae

$\square$ Legionella pneumophila

Influenza virus A

Adenovirus

Respiratory syncytial virus

Undefined

Figure 2. The overall estimated distribution of CAP etiology. $N=154$ in adult publications, $N=115$ in kid publications. 
the most prevalent etiologic agent across age groups, the detection yield was only $15.01 \%$ and $19.16 \%$ in adults and children respectively. The notable explanationwas that majority of reports relied exclusively on serologic testing, which has less specificity than the PCR assay. ${ }^{[11-13]}$ Since M. pneumoniaewas frequently found in mixed infections, multiple simultaneous infections might interfere with the pulmonary cleansing function and thus help establish setting for CAP. ${ }^{[14,15]}$ Atypical pathogens, probably in most cases, caused primary atypical pneumonia and secondary bacterial infections, which had the possibility of missingpreceding pathogenicinsults by clinical practitioners. More specifically in China, some patients arbitrarily received pre-preserved antibiotics such as macrolides or $\beta$-lactams instead of going to the hospital, which could attribute to the bias of low prevalence because all the populations withinthe existing publications were originated from hospital.

S. pneumoniaeremained the leading bacterial cause of both adult and children CAP in our country, followed by Klebsiellapneumoniae, surprisingly, across age groups. Since urinary antigen tests for pneumoncoccus were more sensitive than blood culture and improved the detection of nonbacteremic pneumococcal pathogens with a reported sensitivity of $70 \%$ and a specificity of $90 \%,{ }^{[16-18]}$ they were responsible for the pneumococcal detections in a growing amount of studies. Moreover, the high proportion of S. pneumoniae infection provided a sufficient reason to keep on promotingpneumococcal vaccination especially among children and persons 65 years of age or older. ${ }^{[19]}$ To date, antibiotic resistance in $S$. pneumoniae is still considered to be a nationwide problem. The combined non-susceptibility to penicillin was as high as $55 \%$ in our review, which was higher than the yield reported by CHINET (Chinese network for surveillance of resistant pathogens), accounting for $32.9 \%$ in children and $9.2 \%$ in adults in $2013 .{ }^{[20]}$ This network also reported high resistance to macrolides among S. pneumoniaein China (over 90\% to erythromycin), which is another characteristic difference compared with European/ American countries. Although new fluoroquinolones have good activities against $S$. pneumoniae, the resistance rate of new fluoroquinolones is increasing over time, accounting for up to $15 \%$ to levofloxacin among adults in $2013,{ }^{[20]}$ which is possibly a result of selective pressure due to increased quinolone use in China.

Influenza virus A was the most commonly detected virus among adults. The incidence of influenza virus $A$ was almost four times higher than that among children $(12.28 \%$ vs. $3.73 \%$ ), which underscored the need for improvements in influenza-vaccine uptake and effectiveness ${ }^{[21]}$.Notably, all eligible studies were not specifically conducted during the $2009 \mathrm{H} 1 \mathrm{~N} 1$ influenza pandemic period, which might result in the burden of influenza $A$ less than it would be during seasons with more widespread circulation.On the other hand, similar to results from US, ${ }^{[2]}$ RSV was the most common pathogen detected in children, with the greatest burden observed among children younger than 2 years of age. Given the large proportion and diversity of virus co-infected with other causative agents, all the findings abovesuggested the potential underestimation of the contribution of viruses to pneumonia in our country and highlighted the usefulness of molecular methods for the detection of respiratory virus agents. ${ }^{[2]}$

Our review has certain limitations due to the patchy availability of data. First, most sources of data failed to distinguish the sub-population of adolescents, 15 to 18 years old, from adults, which could bias the epidemiological or etiological findings classified by age. Our review also analyzed few data at $1^{\text {st }}$ or $2^{\text {nd }}$-tier hospital level, which probably represents a high caseload in China. Second, diagnostic methods varied among all the publications, which could have led to underestimation or overestimation of the pathogen-specific rates. Owing to the ethical and feasibility considerations, invasive procedures to obtain specimens directly from the lung were not usually performed, which may have reduced the microbiologic yield. ${ }^{[23]}$ The causal role of sputum or blood cultured bacteria to identify CAP etiologies, however, has also been debated in some cases, particularly in the elderly with comorbidities or children younger than 5 years old since they may not discriminate between infection and colonization. ${ }^{24]}$ Third, in some cases, the clinical and radiographic features of pneumonia overlap with those of other syndromes such as chronic lung disease or congestive heart failure in the elderly and bronchiolitis or reactive airway disease in young children respectively. Even strict definitions may not accurately distinguish among these entities, resulting in potential misclassification. ${ }^{[25]}$ This situation is consistent with the worldwide challenges of diagnosing pneumonia and of its subsequent management.

\section{CONCLUSION}

CAP remained a major public health issue in China, with a relatively higher incidence than that reported in Western countries.Although pathogens were not detected in nearly half of patients, Mycoplasmapneumoniae remained to be the most frequently detected agent across age groups, the detection yield of which was lower than that reported from other countries. Notably, the incidence of influenza virus $A$ in adults was almost four times higher than that inchildrenwhile the prevalence of respiratorysyncytial virus was much less common in adults than that in children.Several concerns should be realized including 1) the influence of immigrants from rural counties seeking for better medical resourcesin relatively developed urban 
areas with a more sophisticated hygienic system, 2) the arbitrary administration of pre-preserved antibiotics such as macrolides or $\beta$-lactams without any recommendation from doctors, 3) the potential underestimation of the proportion of viral pneumonia as well as the usefulness of molecular methods for the detection of respiratory virus agents and 4) the sustainable need to promote pneumococcal vaccination especially among children and persons 65 years of age or older and improve influenzavaccine uptake and effectiveness among adults. Despite some limitations, the value of this review, approaching to systematically review grey published data, is to sketch out the contemporary epidemiological and etiological situation of CAP in our country, which could be useful to help policymakers and clinicians make informed choices and to inspire future studies and surveillance.

\section{Source of Foundation}

This work was supported by grants from excellent youth talent project of Shanghai Municipal commission of health and family planning (No. 2017YQ081) and Shanghai key discipline for respiratory diseases (No. 2017ZZ02014).

\section{Conflict of interest}

We declare that we have no conflict of interest.

\section{REFERENCES}

1. Jain S, Self WH, Wunderink RG, Fakhran S, Balk R, Bramley AM, et al. Community-Acquired Pneumonia Requiring Hospitalization among U.S. Adults. N Engl JMed 2015; 373:415-27.

2. Jain S, Williams DJ, Arnold SR, Ampofo K, Bramley AM, Reed C, et al. Community-acquired pneumonia requiring hospitalization among U.S. children. N Engl JMed 2015; 372:835-45.

3. Bradley JS, Byington CL, Shah SS, Alverson B, Carter ER, Harrison C, et al; the Infectious Diseases Society of A. Executive summary: the management of community-acquired pneumonia in infants and children older than 3 months of age: clinical practice guidelines by the Pediatric Infectious Diseases Society and the Infectious Diseases Society of America. Clin Infect Dis 2011; 53:617-30.

4. Mandell LA, Wunderink RG, Anzueto A, Bartlett JG, Campbell GD, Dean NC, et al; Infectious Diseases Society of A, American Thoracic S. Infectious Diseases Society of America/American Thoracic Society consensus guidelines on the management of community-acquired pneumonia in adults. Clin Infect Dis 2007; 44(Suppl 2):S27-72.

5. Liu Y, Chen M, Zhao T, Wang H, Wang R, Cai B, et al. Causative agent distribution and antibiotic therapy assessment among adult patients with community acquired pneumonia in Chinese urban population. BMC Infect Dis 2009; 9:31.

6. Cao B, Ren LL, Zhao F, Gonzalez R, Song SF, Bai L, et al. Viral and Mycoplasma pneumoniae community-acquired pneumonia and novel clinical outcome evaluation in ambulatory adult patients in China. Eur J Clin Microbiol Infect Dis 2010; 29:1443-8.

7. Shangguan Z, Sun Q, Zhang M, Ding J, Yi L, Gao Y, et al. Mycoplasma pneumoniae infection in hospitalized adult patients with communityacquired pneumonia in China. J Infect Dev Countr 2014; 8:1259-66.
8. Tao LL, Hu BJ, He LX, Wei L, Xie HM, Wang BQ, et al. Etiology and antimicrobial resistance of community-acquired pneumonia in adult patients in China. Chin Med J 2012; 125:2967-72.

9. Huang HH, Zhang YY, Xiu QY, Zhou X, Huang SG, Lu Q, et al. Community-acquired pneumonia in Shanghai, China: microbial etiology and implications for empirical therapy in a prospective study of 389 patients. Eur J Clin Microbiolog Infect Dis 2006; 25:369-74.

10. Moola S, Hagberg L, Churchyard GA, Dylewski JS, Sedani S, Staley H. A multicenter study of grepafloxacin and clarithromycin in the treatment of patients with community-acquired pneumonia. Chest 1999; 116:974-83.

11. Thurman KA, Warner AK, Cowart KC, Benitez AJ, Winchell JM. Detection of Mycoplasma pneumoniae, Chlamydia pneumoniae, and Legionella spp. in clinical specimens using a single-tube multiplex real-time PCR assay. Diagn Micr Infec Dis 2011, 70:1-9.

12. Kumar S, Hammerschlag MR. Acute respiratory infection due to Chlamydia pneumoniae: current status of diagnostic methods. Clin Infect Dis 2007; 44:568-76.

13. Nilsson AC, Bjorkman P, Persson K. Polymerase chain reaction is superior to serology for the diagnosis of acute Mycoplasma pneumoniae infection and reveals a high rate of persistent infection. BMC microbiol 2008; 8:93.

14. Lim WS, Macfarlane JT, Boswell TC, Harrison TG, Rose D, Leinonen $\mathrm{M}$, et al. Study of community acquired pneumonia aetiology (SCAPA) in adults admitted to hospital: implications for management guidelines. Thorax 2001; 56:296-301.

15. Lepow ML, Balassanian N, Emmerich J, Roberts RB, Rosenthal MS, Wolinsky E. Interrelationships of viral, mycoplasmal, and bacterial agents in uncomplicated pneumonia. Am Rev Respir Dis 1968; 97:533-45.

16. Bartlett JG. Diagnostic tests for agents of community-acquired pneumonia. Clin Infect Dis 2011; 52(Suppl 4):S296-304.

17. Murdoch DR, Laing RT, Mills GD, Karalus NC, Town GI, Mirrett S, et al. Evaluation of a rapid immunochromatographic test for detection of Streptococcus pneumoniae antigen in urine samples from adults with community-acquired pneumonia. J Clin Microbiol 2001; 39:3495-8.

18. Werno AM, Murdoch DR. Medical microbiology: laboratory diagnosis of invasive pneumococcal disease. Clin Infect Dis 2008; 46:926-32.

19. Centers for Disease C, Prevention: Use of 13-valent pneumococcal conjugate vaccine and 23-valent pneumococcal polysaccharide vaccine among children aged 6-18 years with immunocompromising conditions: recommendations of the Advisory Committee on Immunization Practices (ACIP). MMWR 2013; 62:521-4.

20. Hu FP, Zhu DM, Wang F, Jiang XF, Sun ZY, Chen ZJ, et al. [CHINET 2013 surveillance of bacterial resistance in China]. Chin J Infect Chemother 2014; 14:369-78.

21. Grohskopf LA, Olsen SJ, Sokolow LZ, Bresee JS, Cox NJ, Broder KR, et al; Centers for Disease C, Prevention. Prevention and control of seasonal influenza with vaccines: recommendations of the Advisory Committee on Immunization Practices (ACIP) -- United States, 2014-15 influenza season. MMWR 2014; 63:691-7.

22. Ruuskanen $\mathrm{O}$, Jarvinen $\mathrm{A}$. What is the real role of respiratory viruses in severe community-acquired pneumonia? Clin Infect Dis 2014; 59:71-3.

23. Karhu J, Ala-Kokko TI, Vuorinen T, Ohtonen P, Syrjala H. Lower respiratory tract virus findings in mechanically ventilated patients with severe community-acquired pneumonia. Clin Infect Dis 2014; 59:62-70.

24. Caliendo AM, Gilbert DN, Ginocchio CC, Hanson KE, May L, Quinn TC, et al; Infectious Diseases Society of A. Better tests, better care: improved diagnostics for infectious diseases. Clin Infect Dis 2013; 57 (Suppl 3):S139-70.

25. Scott JA, Wonodi C, Moisi JC, Deloria-Knoll M, DeLuca AN, Karron RA, et al; Pneumonia Methods Working G. The definition of pneu- 
monia, the assessment of severity, and clinical standardization in the Pneumonia Etiology Research for Child Health study. Clin Infect Dis 2012; 54(Suppl 2):S109-16.
How to cite this article: Zhu Y, Tang $X$, Lu Y, Zhang Y, Qu J. Contemporarysituation of community-acquired pneumonia in China: $A$ systematic review. J Transl Intern Med 2018; 6: 26-31. 